\title{
The protective effect of vitexinin septic encephalopathy by reducing leukocyte-endothelial adhesion and inflammatory response
}

\author{
Haiquan Cao ${ }^{1}$, Xiaojuan Wang ${ }^{2}$, Bing Zhang ${ }^{3}$, Meiping Ren ${ }^{4}$ \\ ${ }^{1}$ Department of Critical Care Medicine, ${ }^{2}$ Department of Endocrinology, ${ }^{3}$ Orthopaedics, The Second Clinical Medical College of North Sichuan \\ Medical College/Nanchong Central Hospital, Nanchong, China; ${ }^{4}$ Department of Pharmacology, Southwest Medical University, Luzhou, China \\ Contributions: (I) Conception and design: H Cao, X Wang; (II) Administrative support: H Cao; (III) Provision of study materials or patients: M Ren, \\ B Zhang, X Wang; (IV) Collection and assembly of data: All authors; (V) Data analysis and interpretation: H Cao, M Ren, B Zhang; (VI) Manuscript \\ writing: All authors; (VII) Final approval of manuscript: All authors. \\ Correspondence to: Haiquan Cao. Department of Critical Care Medicine, The Second Clinical Medical College of North Sichuan Medical College/ \\ Nanchong Central Hospital, Nanchong 637000, China. Email: 3112049007@qq.com.
}

Background: Despite advances in therapeutic strategies and critical care management, septic encephalopathy (SE) is still a leading cause of infection-associated death in intensive care units (ICUs). Vitexin, a flavonoids compound, exerts and anti-inflammatory effect through inhibition of proinflammatory cytokines and signaling pathways. This study aimed to explore the anti-inflammatory effects of vitexin in SE and the underlying mechanisms.

Methods: An SE-inducedC57BL/6 mouse model was established via cecal ligation and puncture (CLP). Western blotting was performed to evaluate the protein expression levels of Chemokine (C-X-C motif) ligand 1 (CXCL1), fractalkine (CX3CL1), intercellular adhesion molecule-1 (ICAM-1), vascular cell adhesion molecule-1 (VCAM-1), E-selectin, NF- $\kappa \mathrm{B}$ p65, p-NF- $\kappa \mathrm{B}$ p65, and tumor necrosis factor- $\alpha$ (TNF- $\alpha$ ). Flow cytometry was used to detect the expressions ofCD11a/CD18, CD11b/CD18, ICAM-1, and adherent leukocyte. The expression of ICAM-1 was detected by immunohistochemistry. An enzyme-linked immunosorbent assay was performed to evaluate the expression of monocyte chemotactic protein-1 (MCP-1), Interleukin (IL)-6, IL-8, and IL-10.

Results: In this study, we found that vitexin significantly downregulated the expression of brain endothelial chemokines CXCL1 and CX3CL1 in CLP mice, exerting a potential anti-inflammatory against SE. Our data also showed that vitexin alleviated SE primarily by relying on reducing leukocyte-endothelial adhesion via the mediation of adhesion molecules. Moreover, vitexin suppressed the expression of proinflammatory cytokines, such as MCP-1, IL-6, IL-8, TNF- $\alpha$, and NF- $\mathrm{BB}$ p65, in the CLP mice, while the expression of the anti-inflammatory cytokine IL-10 was elevated.

Conclusions: Overall, our study demonstrated the protective effect vitexin exerts in SE by reducing leukocyte-endothelial adhesion and inflammatory response. These findings offer a molecular basis for the potential application of vitexin in the treatment of SE and other inflammatory-mediated and immunemediated disorders.

Keywords: Vitexin; leukocyte-endothelial adhesion; inflammatory cytokines; septic encephalopathy (SE)

Submitted Apr 27, 2020. Accepted for publication Jul 04, 2020.

doi: 10.21037/apm-20-1211

View this article at: http://dx.doi.org/10.21037/apm-20-1211 


\section{Introduction}

Septic encephalopathy (SE) is the most common cause of admission to intensive care units (ICUs) and is a leading cause of infection-associated death (1). SE, a cerebral disorder, stems from changes in metabolic and cellular signaling that are mediated by inflammatory components (2). In recent decades, advances in therapeutic strategies and critical care management of SE have been made; however, the currently available therapeutic strategies are still limited and the underlying pathogenic mechanism has yet to be fully illuminated $(3,4)$.

Cell adhesion is a central process in creating a stable environment for cell growth, differentiation, and migration (5). Adhesion molecules are expressed on the cell surface of all tissues, and each hasspecific characteristics. They trigger intracellular pathways and participate in the control of basic physiological processes $(6,7)$. Under normal conditions, the main function of endothelial cells is to maintain the antiadhesive surface by adjusting and suppressing coagulation and restraining leukocyte adhesion $(8,9)$. Many studies have demonstrated that the activation of injured or infected endothelial cells can lead to a decrease in the levels of adhesion molecules and chemokines, causing leukocytes to aggregate to specific sites of inflammation or, immune response (10). Despite the accumulation and activation of endothelial cells being signs of an effective host response to infection, inflammation, and tissue damage (a key step in which is leukocyte adhesion to endothelial cells), leukocyteendothelial interaction may be harmful for the host (11). Studying the adhesion molecules and chemokines that are recruited by leukocytes to endothelial cells may help to understand the molecular regulation of leukocyteendothelial adhesion interactions. E-selectin and P-selectin, for instance, interact with glycosylated ligands; intercellular adhesion molecule-1 (ICAM-1) interacts with lymphocyte function-associated antigen 1; and vascular cell adhesion molecule-1 (VCAM-1) binds to very late antigen $4(12,13)$. Blockade of receptor-ligand interactions serves as the most direct approach to inhibiting leukocyte adhesion to endothelial cells, and provides a potential way to reduce vascular and tissue injury in a variety of inflammatory and immune diseases, including SE (14).

Vitexin, a c-glycosylated flavone, can be isolated from various plants such as pearl millet, hawthorn, and pigeon pea. Recently, owing to its broad pharmacological potential, including anti-tumor, antioxidant, and anti-inflammatory activity, vitexin has drawn a significant amount of attention (15).
He et al. reported that vitexin induced apoptosis by inhibiting autophagy in hepatocellular carcinoma through activating the c-Jun-N-terminal kinase (JNK) signaling pathway (16). Furthermore, other studies have reported that the anti-inflammatory effect exhibited by vitexin largely depends on the inhibition of proinflammatory molecule secretion, including that of monocyte chemotactic protein-1 (MCP-1), Interleukin (IL)-6, and the tumor necrosis factor- $\alpha(\mathrm{TNF}-\alpha)$, as well as an increase in the antiinflammatory cytokine IL-10 (17-20). In this study, the antiinflammatory effects of vitexin on SE were investigated, and the promise of vitexin as a new natural anti-inflammatory candidate for the treatment of SE was evidenced.

We present the following article in accordance with the ARRIVE reporting checklist (available at http://dx.doi. org/10.21037/apm-20-1211).

\section{Methods}

\section{CLP model and drug treatment}

Wild-type C57BL/6 male mice were purchased from Beijing Huafukang Bioscience CO. INC. (Beijing, China). The Committee for Animal Experiments of The Second Clinical Medical School Affiliated to North Sichuan Medical College/Nanchong Central Hospital approved all of the experiments involving animals. This study also conformed to the NIH guidelines for the care and use of animals. CLP was used to induced SE in the mice, and comparisons were made with sham-operated controls. In brief, chloral hydrate (400 $\mathrm{mg} / \mathrm{kg}$ body weight) was administered to anesthetize the mice. A midline incision was made to expose the cecum. Next, the cecum was ligated to the distal end of the ileocecal valve before an 18-gauge needle was used to puncture a hole to cause feces to enter into the enterocoelia (21). The mice were intravenously administered with either vitexin or not. The control group was sham-operated.

\section{Real-time quantitative RT-PCR}

Mouse brain endothelial cells were isolated, as reported previously. Snap-freezing was performed using liquid nitrogen, and the cells were stored at $-80{ }^{\circ} \mathrm{C}$ until use (22). A TRIzol reagent kit (Invitrogen, Beijing, China) was used to isolate total RNA, following the manufacturer's protocol. PrimeScript RT reagent Kit (TakaRa, Dalian, China) was used to carry out reverse transcription following the manufacturer's protocol. The 2 SYBR Premix Ex 
Taq $^{\text {TM }}$ II (TakaRa, Dalian, China) was used to assemble the quantitative real time-polymerase chain reactions (qRTPCRs), which were subjected in Bio-Rad CFX-96 (BioRad, CA, USA) to the protocol as follows: $30 \mathrm{~s}$ at $95^{\circ} \mathrm{C}$, 40 cycles of $10 \mathrm{~s}$ at $95{ }^{\circ} \mathrm{C}, 30 \mathrm{~s}$ at $60{ }^{\circ} \mathrm{C}$ and $30 \mathrm{~s}$ at $72{ }^{\circ} \mathrm{C}$. The melting curve was obtained using temperatures from 65 to $95{ }^{\circ} \mathrm{C}$ in $1{ }^{\circ} \mathrm{C} / 10 \mathrm{~s}$ increments. Glyceraldehyde 3 -phosphate dehydrogenase (GAPDH) was used for normalization. The $2^{-\Delta \Delta \mathrm{Ct}}$ method was applied to analyze relative changes in the levels of mRNA expression (23). The primer sequences used were as follows: Chemokine (C-X-C motif) ligand 1 (CXCL1), forward primer CGTTGACATCCGTAAAGACC, reverse primer AACAGTCCGCCTAGAAGCAC; fractalkine (CX3CL1), forward primer CTACTAGGAGCTGCGACACG, reverse primer AAG-CCACTGGGATTCGTGAG; glyceraldehyde-3-phosphate dehydrogenase (GAPDH), forward primer CGGAGTCAACG- GATTTGGTCGTAT, reverse primer AGCCTTCTCCATGGTGGTGAAGAC.

\section{Western blot}

The protein extracted from the mouse brain endothelial cells was used for western blotting. RIPA lysis buffer (Beyotime Institute of Biotechnology, Shanghai, China) was used to extract cellular proteins following the instructions of the manufacturer. The BCA Protein Assay Kit was employed to detect the protein concentrations. Next, protein separation was performed with $10 \%$ dodecyl sulfate polyacrylamide gel electrophoresis (SDS-PAGE). Following that, electroblotting was carried out to transfer the separated proteins to polyvinylidene difluoride (PVDF) membranes (Merck Millipore). Then, blocking with 5\% skim milk in Tris-buffered saline Tween (TBST) was performed for $1 \mathrm{~h}$. The following antibodies were used: anti-CXCL1 (ab9955, 1:1,000, Abcam), anti-CX3CL1 (ab25088, 1:1,000, Abcam), anti-ICAM-1, (ab109361, 1:1,000, Abcam), anti-VCAM-1, (ab134047, 1:2,000, Abcam), anti-E-selectin (ab2497, 1:1,000, Abcam), anti-NF-кB p65(ab16502, 1:1,000, Abcam), anti-p-NF-кB p65 (ab86299, 1:2,000, Abcam), and TNF- $\alpha$ (ab1973, 1:1,000, Abcam), and anti-GAPDH (Zenbio, Chengdu, China).

\section{Flow cytometry}

Before the mice were decapitated, peripheral blood samples were obtained for flow cytometry analysis, as previously reported (24). First, $100 \mu \mathrm{L}$ of whole blood were gently mixed with $10 \mu \mathrm{L}$ of anti-mouse mAbs (PE-anti mouse CD11a/CD18, PE-anti mouse CD11b/CD18, and PEanti mouse ICAM-1). The mixture was then incubated for $20 \mathrm{~min}$ at room temperature in the dark.

\section{Immunobistochemistry}

The mice were anesthetized and via transcardial perfusion, fixed with 4\% paraformaldehyde in phosphate-buffered saline (PBS). After that, the brains were wholly fixed with $4 \%$ paraformaldehyde for $24 \mathrm{~h}$, then embedded in paraffin and sectioned. Xylene was used to separate the paraffinized sections before rehydration with gradient ethanol. Next, $10 \mathrm{mM}$ citric acid buffer was used to extract the antigen, after which the tissue sections were kept in an incubator with 3\% $\mathrm{H}_{2} \mathrm{O}_{2}$ for $10 \mathrm{~min}$ and then sealed for $1 \mathrm{~h}$ at room temperature. The heart tissue sections were incubated overnight with an anti-ICAM-1 antibody (Abcam, Cambridge, UK), after which they were exposed to fluorescent secondary antibodies following the instructions of the manufacturer. The corresponding secondary antibody was incubated at room temperature for $1 \mathrm{~h}$. Finally, an Olympus DX51 fluorescence microscope (Olympus, Tokyo, Japan) was used to observe the images, and data analysis was performed using Image-Pro Plus 6.0 (Media Cybernetics, USA).

\section{Enzyme-linked immunosorbent assay (ELISA) assay}

The ELISA assay was used to quantify the levels of MCP1, IL-6, IL-8, and IL-10 protein. The total protein in the cell lysate extracted from the peripheral blood of the mice was measured with the QuantiPro ${ }^{\text {TM }}$ BCA Assay Kit (Sigma, The Netherlands) following the manufacturer's instructions. The DuoSet ELISA Development kit (R\&D) was employed to measure the protein expressive levels of MCP-1, IL-6, IL-8, and IL-10 in the cell lysates following the protocol of the manufacturer. The protein content was normalized by total protein.

\section{Statistical analysis}

Each experiment in this study was carried out in triplicate, and the data were expressed as mean \pm SD. Statistical analyses were conducted using IBM SPSS Statistics 25.0. (IBM, Armonk, USA). The differences between the two groups were analyzed by Student's $t$-test. The significance of differences between treatment groups was analyzed using one-way analysis of variance (ANOVA). $\mathrm{P}<0.05$ was 
<smiles>O=c1cc(-c2ccc(O)cc2)oc2c(C3O[C@H](CO)[C@@H](O)[C@H](O)[C@H]3O)c(O)cc(O)c12</smiles>

$\mathrm{C} 21 \mathrm{H} 20 \mathrm{O} 10$ $\mathrm{MW}: 432.38$
B
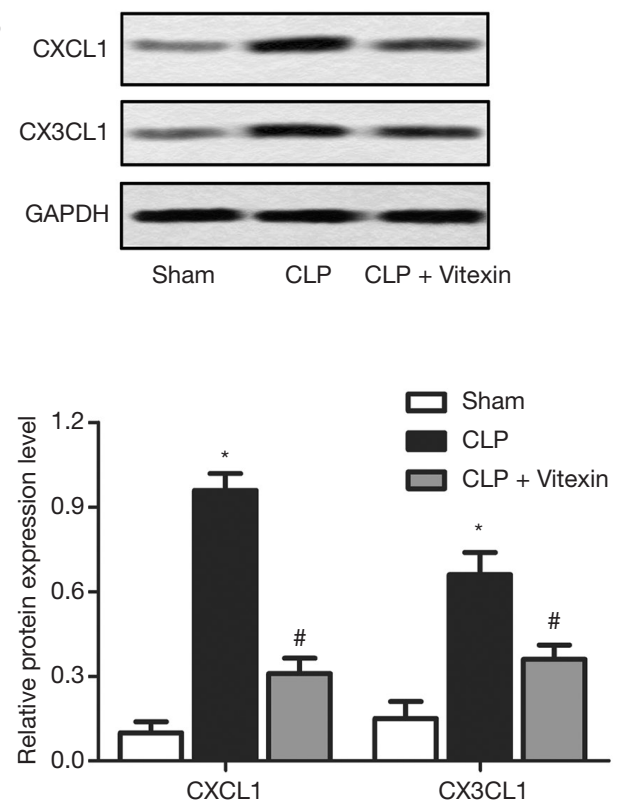

Figure 1 Effect of vitexin on the brain endothelial chemokine expression. (A) The structure of vitexin. (B) The expression of CXCL1 and CX3CL1 was evaluated by western blotting. The histogram shows the relative expression of the two proteins. Data are expressed as the mean $\pm \mathrm{SD}$, from three independent experiments. *, $\mathrm{P}<0.05$ vs. the control group; ${ }^{\#}, \mathrm{P}<0.05$ vs. the CLP group.

considered to show statistical significance.

\section{Results}

\section{Vitexin downregulates the expression of brain endothelial chemokines}

Septic injury has been reported to increase the expression levels of chemokines CXCL1 and CX3CL1 in brain endothelial cells (14). Western blotting revealed that the expression levels of CXCL1 and CX3CL1 were significantly higher in the mice with CLP-induced sepsis than in the control group (Figure 1). Moreover, after treatment with vitexin, the expression levels of CXCL1 and CX3CL1 were significantly lower than CLP-induced sepsis group (Figure 1). These results suggested that vitexin might have protective effects on SE.

\section{Vitexin inbibits leukocyte adhesion to endothelial cells}

$\beta 2$ integrins are the main cell surface adhesion molecules expressed in leukocytes and are responsible for mediating leukocytes adhesion to endothelial cells through binding to ICAM-1 (25). This study demonstrated that the leukocytic expression of CD11a/CD18 and CD11b/CD18 in the peripheral blood of the mice detected by flow cytometry was increased by CLP-induced sepsis, while vitexin successfully neutralized this rise (Figure 2A,B). Meanwhile, leukocytic expression of ICAM-1 was activated after CLP, while this activation was inhibited by vitexin treatment (Figure 2C).

Several studies have reported that ICAM-1, VCAM1 , and E-selectin to be endothelial adhesion proteins that are transcriptionally regulated by cytokines or other inflammatory mediators (13). Our results showed that the protein expression levels of ICAM-1, VCAM-1, and E-selectin were elevated by CLP-induced sepsis, while these effects successfully alleviated by vitexin (Figure $3 A, B, C, D$ ). The results of immunohistochemistry also confirmed that vitexin held the potential to neutralize the increased expression level of ICAM-1 in the CLP mice (Figure 3E). Moreover, the treatment of vitexin significantly alleviated the increased number of adherent leukocytes to CLPinduced brain vessels (Figure 3F). These data demonstrated that vitexin may suppress leukocyte-endothelial adhesion through the inhibition of the $\beta 2$ integrin/ICAM- 1 signaling pathway and endothelial adhesion protein expression.

\section{Vitexin mediates inflammatory cytokine expression}

Previous studies have reported that the levels of 
A
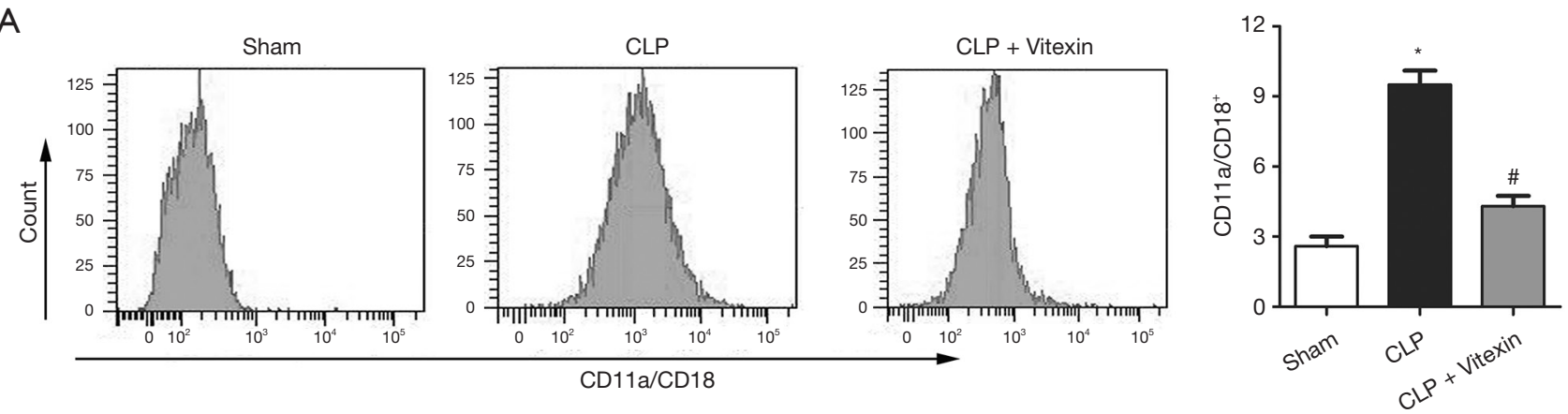

B
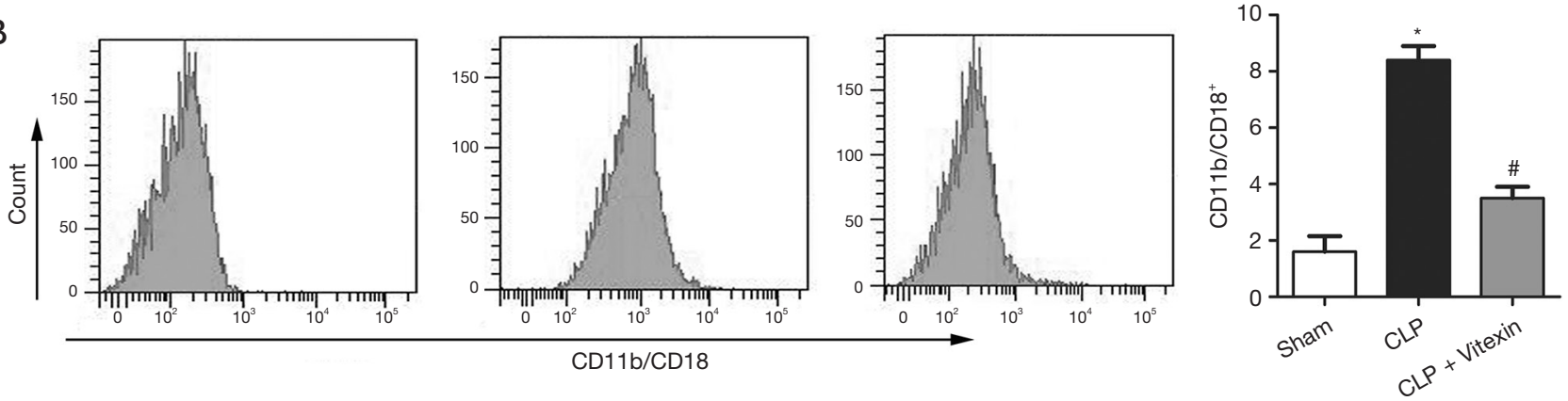

C
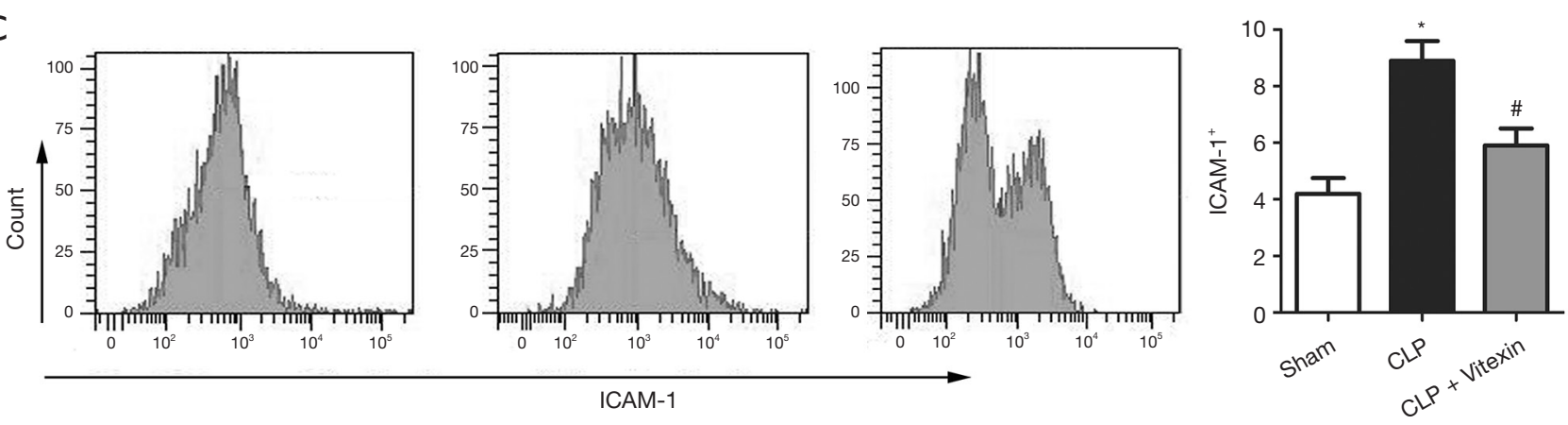

Figure 2 Effect of vitexin on $\beta 2$ integrin/ICAM-1 pathway. The expression of CD11a/CD18 (A), CD11b/CD18 (B), and ICAM-1 (C) was detected by flow cytometry. Data are expressed as the mean $\pm \mathrm{SD}$, from three independent experiments. *, $\mathrm{P}<0.05 v s$. the control group; *, $\mathrm{P}<0.05$ vs. the CLP group.

proinflammatory cytokines are highly elevated in the peripheral blood plasma of sepsis patients $(26,27)$. In line with the findings of the previous studies, our data demonstrated that the expression of MCP-1, IL-6, and IL-8 was significantly upregulated by CLP-induced sepsis, while vitexin was able to alleviate these rises (Figure $4 A, B, C$ ). Moreover, after treatment with vitexin, the expression level of IL-10was increased in comparison with the CLP group (Figure 4D). These results suggested that vitexin could simultaneously reduce proinflammatory cytokine expression and increase anti-inflammatory cytokine expression.

\section{Vitexin suppresses $p-N F-\kappa B$ p65 and TNF- $\alpha$ activation}

In the pathophysiology of sepsis, NF- $\mathrm{\kappa B}$ p65 plays a key role in proinflammatory gene transcription (28). TNF- $\alpha$ also acts as a principal activator of the inflammatory cascade (29). The present data demonstrated that the expression levels of p-p65/p-65 and TNF- $\alpha$ were significantly elevated by CLP-induced sepsis, while vitexin could significantly alleviate these increases (Figure $5 A, B, C$ ). These data suggested that the protective effects on sepsis might attributed in part to the inhibition of p-p65/p-65 and TNF- $\alpha$ by vitexin. 


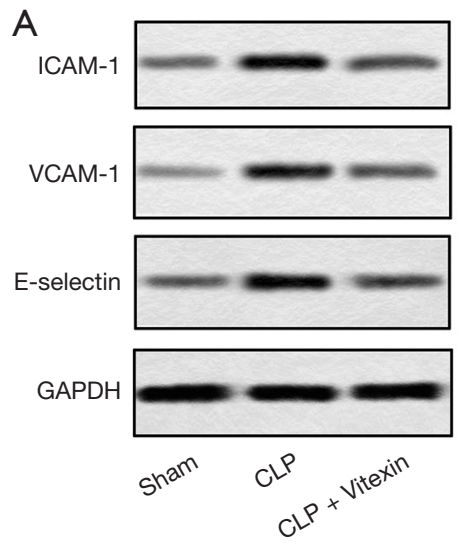

$\mathrm{E}$

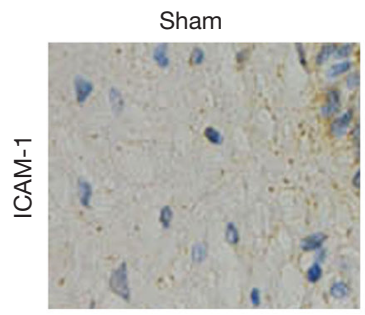

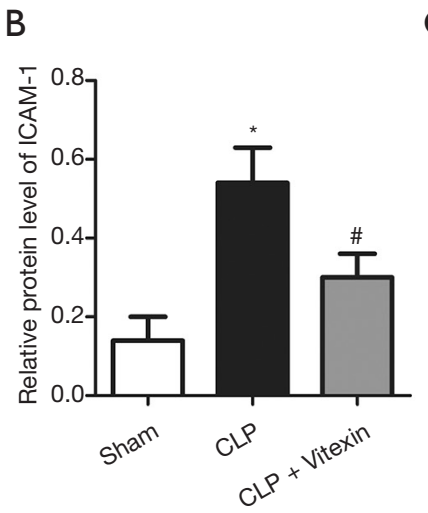
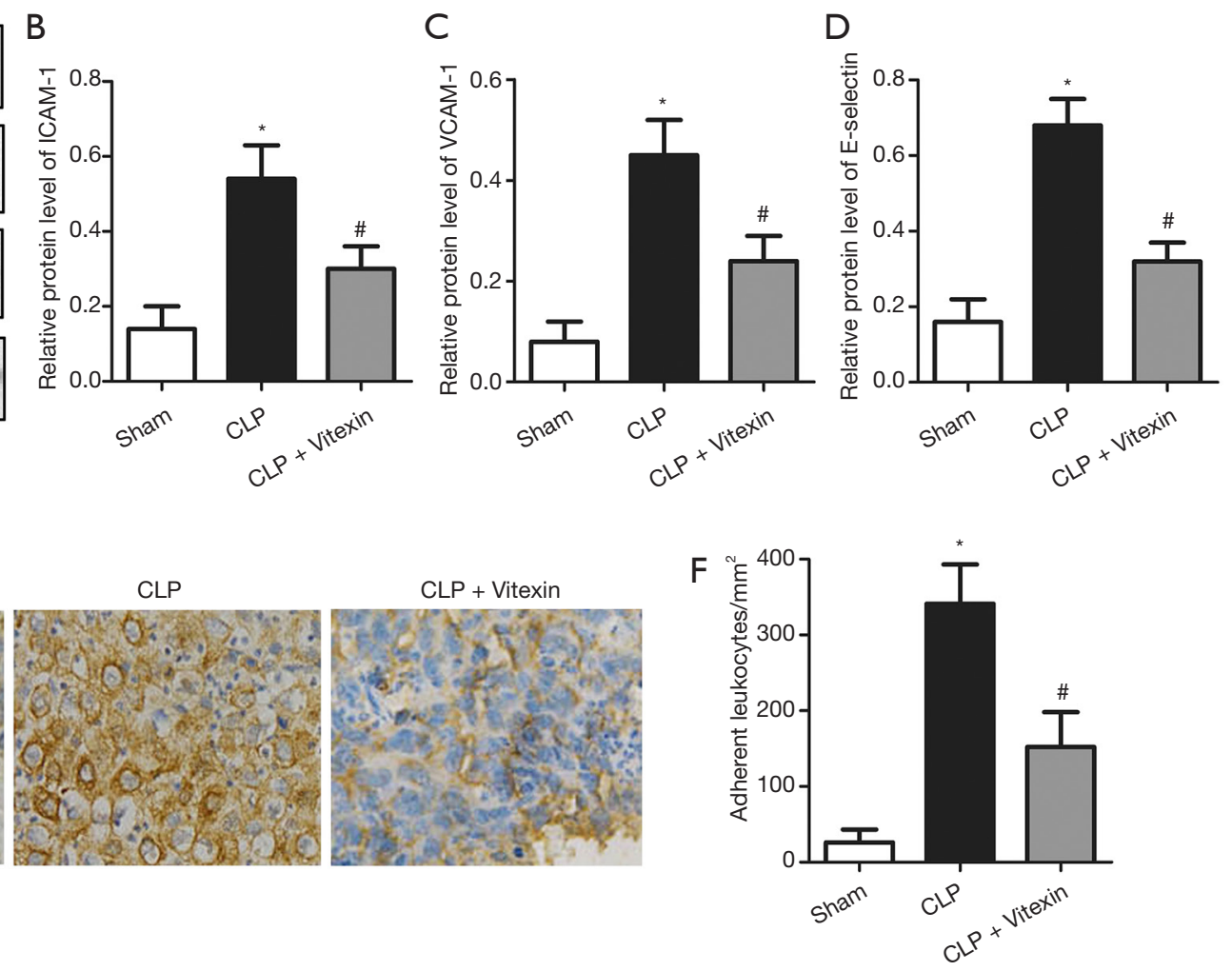

Figure 3 Effect of vitexin on endothelial adhesion molecule expression. (A) The expression of ICAM-1, VCAM-1, and E-selectin was evaluated by western blotting. The histogram shows the relative expression of ICAM-1 (B), VCAM-1 (C), and E-selectin (D). (E) The expression of ICAM-1 was detected by immunohistochemistry. Magnification, 400×. (F) The expression of adherent leukocytes was detected by flow cytometry. Data are expressed as the mean $\pm \mathrm{SD}$, from three independent experiments. *, $\mathrm{P}<0.05$ vs. the control group; ${ }^{*}, \mathrm{P}<0.05$ vs. the CLP group.
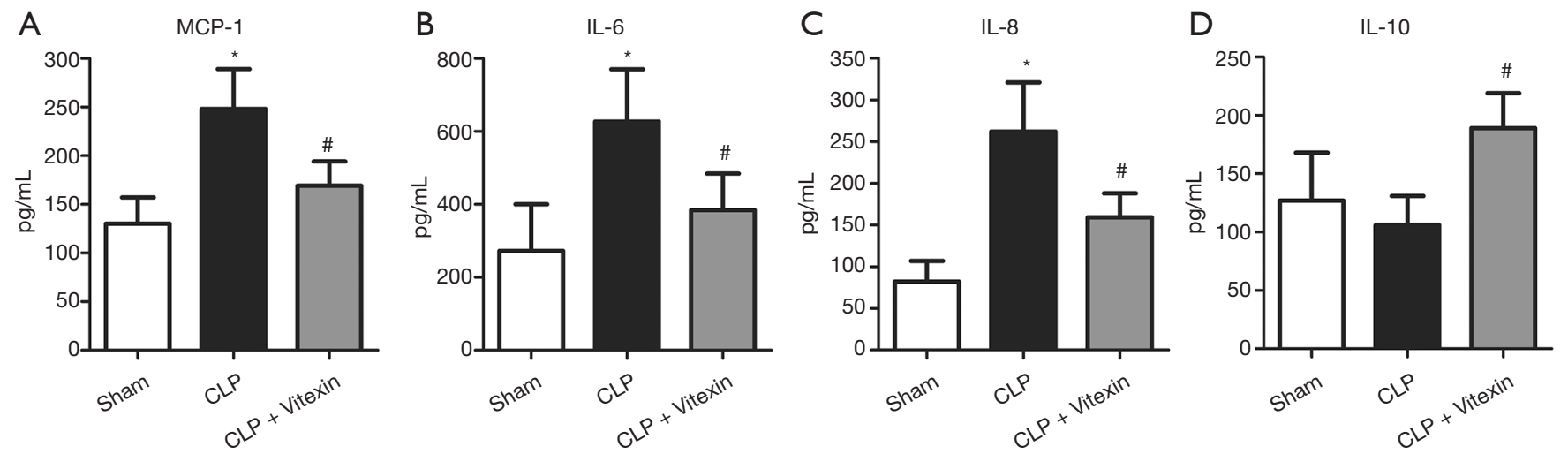

Figure 4 Effect of vitexin on pro-/anti-inflammatory cytokine expression. The expression of MCP-1 (A), IL-6 (B), IL-8 (C) and IL-10 (D) was evaluated by ELISA. Data are expressed as the mean $\pm \mathrm{SD}$, from three independent experiments. * $\mathrm{P}<0.05$ vs. the control group; , $\mathrm{P}<0.05$ vs. the CLP group. ELISA, enzyme-linked immunosorbent assay. 

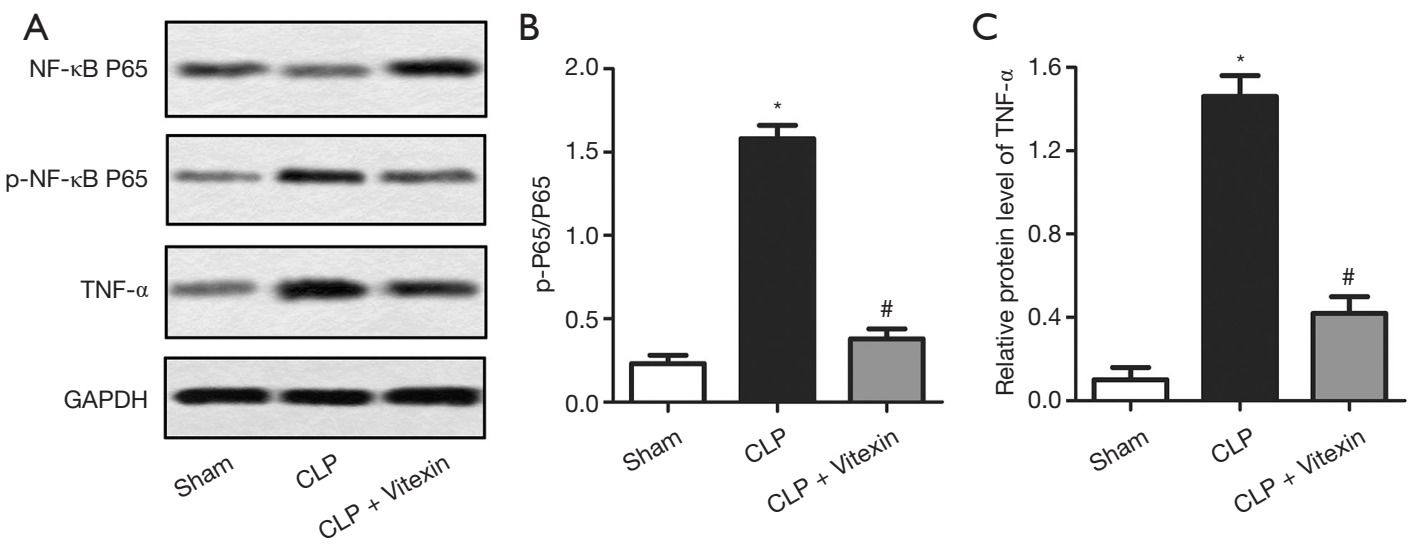

Figure 5 Effect of vitexin on $\mathrm{p}-\mathrm{NF}-\kappa \mathrm{B}$ p65 and TNF- $\alpha$ activation. (A) The expression of NF- $\kappa \mathrm{B}$ p65, p-NF- $\kappa \mathrm{B}$ p65, and TNF- $\alpha$ was evaluated by western blotting. The histogram shows the relative expression of p-p65/p65 (B) and TNF- $\alpha$ (C). Data are expressed as the mean $\pm \mathrm{SD}$, from three independent experiments. * $\mathrm{P}<0.05$ vs. the control group; ${ }^{\#}, \mathrm{P}<0.05$ vs. the CLP group. TNF- $\alpha$, tumor necrosis factor- $\alpha$.

\section{Discussion}

Natural compounds can be valuable resources for the prevention and treatment of various diseases, including sepsis. Honokiol, which is derived from Magnolia officinalis, has been showed to possess anti-inflammatory and antioxidant properties. A study by Li revealed that honokiol could antagonize sepsis-associated acute kidney injury partially through blocking the NF- $\mathrm{KB}$ signaling pathway (30). The progress of septic shock can be reversed by $\alpha$-iso-cubebenol, which triggers numerous protective downstream signaling pathways, enhancing microbial killing and maintaining organ function and leukocyte survival (31). Meanwhile, wild bitter gourd can reduce fat accumulation and improve low blood glucose in sepsis, as well as reduce inflammatory markers in mice with sepsis (32). Recently, vitexin has been investigated for its broad variety of pharmacological effects, including anti-cancer, anti-oxidant and anti-inflammatory properties (15). For instance, vitexin was shown to trigger apoptosis in human esophageal cancer cells EC-109, leukemia cells U937, and oral cancer cells OC2, exerting its potential against tumor growth (33-35). Furthermore, vitexin significantly reduced the levels of TNF- $\alpha$ and IL-17 in pleural fluid leakage in a carrageenaninduced pleurisy mouse model, and it has exhibited an analgesic ability in several inflammatory pain animal models $(17,36)$. These results suggest that vitexin may hold potential for treating diseases involving inflammation. Among its variety of attributes, the effect vitexin has on inflammation has drawn considerable attention, possibly because of its ability to regulate proinflammatory/antiinflammatory cytokines and signaling pathways, such as IL6, IL-8, IL-10, TNF- $\alpha$, and NF- $\mathrm{kB}$ (15). Despite the many existing possibilities regarding the therapeutic potential of vitexin, its mechanisms deserve more systematic study, which may eventually contribute to the pharmacotherapy of various disease conditions. Here, we set out to investigate the therapeutic effects of vitexin in SE as well as the underlying molecular and cellular mechanisms associated with these effects, and to obtain experiment-based evidence of the possible value of vitexin as a novel natural antiinflammatory candidate for the treatment of SE.

Leukocyte-endothelial cell adhesion is a crucial event in host defense and tissue damage repair; however, in some pathological conditions, this interaction may lead to organ dysfunction and tissue and vascular damage. Many studies have demonstrated that blockade of leukocyte adhesion to endothelium molecules has the potential to combat inflammatory-mediated and immune-mediated injury in various diseases (10). Simvastatin, for instance, showed a potent and effective endothelium-protective ability that reduced leukocyte-endothelial cell adhesion in rats, which suggests it has anti-inflammatory potential besides its wellknown lipid-lowering effect (37). Targeting formyl peptide receptor 2 reduced leukocyte-endothelial cell adhesion in a murine stroke model and exerted a potential effect against excess inflammation (38). Moreover, sarpogrelate hydrochloride was shown to inhibit vascular inflammation in obesity, possibly by inactivating leukocyte adhesion to 
endothelial cells (39). Recently, a number of studies have investigated the molecular events associated with leukocyteendothelial cell adhesion that contribute to the development of SE, which is a critical determining factor of sepsisrelated mortality (14,40-42). These results support the idea that leukocyte-endothelial cell adhesion serves a role in linking vascular inflammation to brain damage, and provide rationale therapeutic strategies for the treatment of SE.

CXC chemokines play a crucial role in leukocyte activation and subsequently promote their adhesion to endothelial cells (43). In a previous study, Roy et al. reported that, during encephalomyelitis, leukocyte-endothelial cell adhesion induced CXCL1 family cytokines in the brain (44). Furthermore, A research paper by Wang et al. further reported a significant increase in CXCL1 and CX3CL1 expression in brain microvessels during sepsis (14). Our findings are consistent with those of earlier studies and show that CXCL1 and CX3CL1 expression was increased in CLP-induced sepsis. More importantly, the data showed that vitexin was able to significantly decrease the expression levels of both CXCL1 and CX3CL1 in CLP-induced sepsis compared with the non-treated group, indicating that vitexin might hold potential for the treatment of SE.

To date, of the integrin adhesion receptor family, only five members have been shown to have involvement in the adhesion of leukocytes to endothelial cells, including $\beta 2$ leukocyte integrin (CD11a/CD18, CD11b/CD18, and CD11c/CD18) and $\beta 1$ integrin proteins VLA-4 $(\alpha 4 \beta 1$, CD49d/CD29) and $\alpha 4 \beta 7$. Leukocyte adhesion to endothelial cells relies primarily on CD11a/CD18 and CD11b/CD18 binding to endothelial ligand ICAM-1 $(9,13)$. In addition, previous research has demonstrated that CXCL1 promotes endothelial activation and leukocyte adhesion via the $\beta 2$ integrin/ICAM-1 pathway (14). Consistent with above results, our data shows that the levels of CD11a/CD18, CD11b/CD18 and ICAM-1 in the peripheral blood of the mice were increased by CLP-induced sepsis, while vitexin was able to neutralize these increases. These data suggest that intexin may possess the ability to inhibit leukocyte adhesion to endothelial cells. ICAM-1, VCAM-1, and E-selectin are endothelial adhesion proteins, which can be regulated by cytokines or other inflammatory mediators (13). Kim et al. reported that by decreasing the expression levels of these three endothelial adhesion proteins, leukocyte adhesion to endothelial cells could be reduced (45). In the present study, vitexin significantly reduced the expression of these adhesion proteins and also had a down-regulatory effect on adherent leukocytes, which indicates that vitexin exerts its protective effects on SE partially through the inhibition of leukocyte-endothelium adhesion.

The release of cytokines into the circulation is the central to the early development and persistence of sepsis. Proinflammatory molecules including MCP-1, IL-6, and IL-8, and anti-inflammatory molecules, such as IL10 , are always involved in inflammatory and immune reactions, exerting totally opposite effects $(46,47)$. NF$\kappa \mathrm{B}$ is a general transcription factor involved in regulating the gene expression of cytokines and inflammationrelated genes. Inhibiting the excessive activation of NF$\kappa \mathrm{B}$ is an effective way of controlling inflammation-related diseases, like sepsis. A study by Park et al. showed that inhibition of NF- $\kappa \mathrm{B}$ suppressed severe LPS-induced sepsis (48). The proinflammatory cytokine TNF- $\alpha$ has a crucial responsibility in cytokine cascade activation, which macrophages release in response to infection, and stimulating the production of downstream cytokines such as IL-6 and IL-8. Previous research has demonstrated that in sepsis and septic shock models, elevated levels of TNF- $\alpha$ are associated with mortality (49). In this study, all of the above proinflammatory cytokines were increased in the CLP mice, which is consistent with previous research. More importantly, vitexin could significantly neutralize their harmful effects and also exerted a protective effect in SE.

Overall, our study suggests that vitexin effectively alleviates SE by inhibiting leukocyte-endothelium adhesion and proinflammatory cytokine expression. However, the pathogenesis of sepsis-associated encephalopathy (SAE) is multifactorial, involving neurotransmitter alterations, inflammatory cytokines, oxidative damage, mitochondrial dysfunction, apoptosis, and other factors. Importantly, oxidative stress may promote the pathogenesis of SAE by enhancing the expression of proinflammatory cytokines, promoting apoptosis, and/or interfering with blood-brain barrier function (50,51). Early sepsis is associated with a decrease in mitochondrial ATP generation, which is likely mediated by cytokines, reactive oxygen species (ROS), and nitric oxide (NO) (52). From the molecular structure of vitamin, it can be found that vitexin has strong antioxidant properties. Importantly, previous research has exhibited that Vitexin is an active component from medicinal plants which has antioxidant and anti-inflammatory activities. Vitexin exhibits an analgesic effect in a variety of inflammatory pain models by targeting TRPV1 and oxidative stress and by modulating cytokine production (53). The inhibiting role of Vitexin on NLRP3 inflammasome may via Nrf2mediated reduction of ROS production (54). So whether 
the anti-inflammatory function of vitexin works through antioxidants. This requires us to do further experiments to verify the pharmacological mechanism of vitexin.

\section{Acknowledgments}

Funding: None.

\section{Footnote}

Reporting Checklist: The authors have completed the ARRIVE reporting checklist. Available at http://dx.doi. org/10.21037/apm-20-1211

Data Sharing Statement: Available at http://dx.doi. org/10.21037/apm-20-1211

Conflicts of Interest: All authors have completed the ICMJE uniform disclosure form (available at http://dx.doi. org/10.21037/apm-20-1211). The authors have no conflicts of interest to declare.

Ethical Statement: The authors are accountable for all aspects of the work in ensuring that questions related to the accuracy or integrity of any part of the work are appropriately investigated and resolved. The Committee for Animal Experiments of The Second Clinical Medical School Affiliated to North Sichuan Medical College/ Nanchong Central Hospital approved all of the experiments involving animals (No. 2017-44). This study also conformed to the NIH guidelines for the care and use of animals.

Open Access Statement: This is an Open Access article distributed in accordance with the Creative Commons Attribution-NonCommercial-NoDerivs 4.0 International License (CC BY-NC-ND 4.0), which permits the noncommercial replication and distribution of the article with the strict proviso that no changes or edits are made and the original work is properly cited (including links to both the formal publication through the relevant DOI and the license). See: https://creativecommons.org/licenses/by-nc-nd/4.0/.

\section{References}

1. Mittal MK, Kashyap R, Herasevich V, et al. Do patients in a medical or surgical ICU benefit from a neurologic consultation? Int J Neurosci 2015;125:512-20.

2. Dal-Pizzol F, Tomasi CD, Ritter C. Septic encephalopathy: does inflammation drive the brain crazy? Rev Bras Psiquiatr 2014;36:251-8.

3. Gofton TE, Young GB. Sepsis-associated encephalopathy. Nat Rev Neurol 2012;8:557-66.

4. Ziaja M. Septic encephalopathy. Curr Neurol Neurosci Rep 2013;13:383.

5. Ruoslahti E, Pierschbacher MD. New perspectives in cell adhesion: RGD and integrins. Science 1987;238:491-7.

6. Edelman GM. Cell adhesion molecules. Science 1983;219:450-7.

7. Cunningham BA, Hemperly JJ, Murray BA, et al. Neural cell adhesion molecule: structure, immunoglobulin-like domains, cell surface modulation, and alternative RNA splicing. Science 1987;236:799-806.

8. Key NS. Scratching the surface: endothelium as a regulator of thrombosis, fibrinolysis, and inflammation. J Lab Clin Med 1992;120:184-6.

9. Bevilacqua MP, Nelson RM, Mannori G, et al. Endothelial-leukocyte adhesion molecules in human disease. Annu Rev Med 1994;45:361-78.

10. Harlan JM, Winn RK. Leukocyte-endothelial interactions: clinical trials of anti-adhesion therapy. Crit Care Med 2002;30:S214-9.

11. Zahr A, Alcaide P, Yang J, et al. Endomucin prevents leukocyte-endothelial cell adhesion and has a critical role under resting and inflammatory conditions. Nat Commun 2016;7:10363.

12. Dieterich LC, Huang H, Massena S, et al. alphaBcrystallin/HspB5 regulates endothelial-leukocyte interactions by enhancing NF-kappaB-induced upregulation of adhesion molecules ICAM-1, VCAM-1 and E-selectin. Angiogenesis 2013;16:975-83.

13. Carlos TM, Harlan JM. Leukocyte-endothelial adhesion molecules. Blood 1994;84:2068-101.

14. Wang H, Hong LJ, Huang JY, et al. P2RX7 sensitizes Mac-1/ICAM-1-dependent leukocyte-endothelial adhesion and promotes neurovascular injury during septic encephalopathy. Cell Res 2015;25:674-90.

15. He M, Min JW, Kong WL, et al. A review on the pharmacological effects of vitexin and isvitexin. Fitoterapia 2016;115:74-85.

16. He JD, Wang Z, Li SP, et al. Vitexin suppresses autophagy to induce apoptosis in hepatocellular carcinoma via activation of the JNK signaling pathway. Oncotarget 2016;7:84520-32.

17. Borghi SM, Carvalho TT, Staurengo-Ferrari L, et al. Vitexin inhibits inflammatory pain in mice by targeting TRPV1, oxidative stress, and cytokines. J Nat Prod 
2013;76:1141-9.

18. Kang I, Choi S, Ha TJ, et al. Effects of Mung Bean (Vigna radiata L.) Ethanol Extracts Decrease Proinflammatory Cytokine-Induced Lipogenesis in the KK-Ay Diabese Mouse Model. J Med Food 2015;18:841-9.

19. Dos Reis GO, Vicente G, de Carvalho FK, et al. Croton antisyphiliticus Mart. attenuates the inflammatory response to carrageenan-induced pleurisy in mice. Inflammopharmacology 2014;22:115-26.

20. Lin CM, Huang ST, Liang YC, et al. Isovitexin suppresses lipopolysaccharide-mediated inducible nitric oxide synthase through inhibition of NF-kappa B in mouse macrophages. Planta Med 2005;71:748-53.

21. Rittirsch D, Huber-Lang MS, Flierl MA, et al. Immunodesign of experimental sepsis by cecal ligation and puncture. Nat Protoc 2009;4:31-6.

22. Howland SW, Gun SY, Claser C, et al. Measuring antigen presentation in mouse brain endothelial cells ex vivo and in vitro. Nat Protoc 2015;10:2016-26.

23. Teng X, Zhang Z, He G, et al. Validation of reference genes for quantitative expression analysis by real-time RTPCR in four lepidopteran insects. J Insect Sci 2012;12:60.

24. Huyan XH, Lin YP, Gao T, et al. Immunosuppressive effect of cyclophosphamide on white blood cells and lymphocyte subpopulations from peripheral blood of Balb/ c mice. Int Immunopharmacol 2011;11:1293-7.

25. Xu J, Gao XP, Ramchandran R, et al. Nonmuscle myosin light-chain kinase mediates neutrophil transmigration in sepsis-induced lung inflammation by activating 2 integrins. Nat Immunol 2008;9:880-6.

26. Sugitharini V, Prema A, BerlaThangam E. Inflammatory mediators of systemic inflammation in neonatal sepsis. Inflamm Res 2013;62:1025-34.

27. Schwager J, Richard N, Mussler B, et al. Tomato Aqueous Extract Modulates the Inflammatory Profile of Immune Cells and Endothelial Cells. Molecules 2016;21:168.

28. Courtine E, Pene F, Cagnard N, et al. Critical role of cRel subunit of NF-kappaB in sepsis survival. Infect Immun 2011;79:1848-54.

29. Lv S, Han M, Yi R, et al. Anti-TNF-alpha therapy for patients with sepsis: a systematic meta-analysis. Int J Clin Pract 2014;68:520-8.

30. Li N, Xie H, Li L, et al. Effects of honokiol on sepsisinduced acute kidney injury in an experimental model of sepsis in rats. Inflammation 2014;37:1191-9.

31. Lee SK, Kim SD, Kook M, et al. Therapeutic effects of alpha-iso-cubebenol, a natural compound isolated from the Schisandra chinensis fruit, against sepsis. Biochem Biophys
Res Communi 2012;427:547-52.

32. Chao CY, Sung PJ, Wang WH, et al. Anti-inflammatory effect of Momordica charantia in sepsis mice. Molecules 2014;19:12777-88.

33. An F, Wang S, Tian Q, et al. Effects of orientin and vitexin from Trolliuschinensis on the growth and apoptosis of esophageal cancer EC-109 cells. Oncol Lett 2015;10:2627-33.

34. Lee CY, Chien YS, Chiu TH, et al. Apoptosis triggered by vitexin in U937 human leukemia cells via a mitochondrial signaling pathway. Oncol Rep 2012;28:1883-8.

35. Yang SH, Liao PH, Pan YF, et al. The novel p53dependent metastatic and apoptotic pathway induced by vitexin in human oral cancer OC2 cells. Phytother Res 2013;27:1154-61.

36. Dong L, Fan Y, Shao X, et al. Vitexin protects against myocardial ischemia/reperfusion injury in Langendorffperfused rat hearts by attenuating inflammatory response and apoptosis. Food Chem Toxicol 2011;49:3211-6.

37. Pruefer D, Scalia R, Lefer AM. Simvastatin inhibits leukocyte-endothelial cell interactions and protects against inflammatory processes in normocholesterolemic rats. Arterioscler Thromb Vasc Biol 1999;19:2894-900.

38. Smith HK, Gil CD, Oliani SM, et al. Targeting formyl peptide receptor 2 reduces leukocyte-endothelial interactions in a murine model of stroke. FASEB J 2015;29:2161-71.

39. Kataoka H, Ariyama Y, Deushi M, et al. Inhibitory Effect of Serotonin Antagonist on LeukocyteEndothelial Interactions In Vivo and In Vitro. PLoS One 2016;11:e0147929.

40. Ferri LE, Swartz D, Christou NV. Soluble L-selectin at levels present in septic patients diminishes leukocyteendothelial cell interactions in mice in vivo: a mechanism for decreased leukocyte delivery to remote sites in sepsis. Crit Care Med 2001;29:117-22.

41. van Griensven M, Probst C, Muller K, et al. Leukocyteendothelial interactions via ICAM-1 are detrimental in polymicrobial sepsis. Shock 2006;25:254-9.

42. Joyce DE, Nelson DR, Grinnell BW. Leukocyte and endothelial cell interactions in sepsis: relevance of the protein C pathway. Crit Care Med 2004;32:S280-6.

43. Zhang S, Hwaiz R, Rahman M, et al. Ras regulates alveolar macrophage formation of CXC chemokines and neutrophil activation in streptococcal M1 protein-induced lung injury. Eur J Pharmacol 2014;733:45-53.

44. Roy M, Richard JF, Dumas A, et al. CXCL1 can be regulated by IL-6 and promotes granulocyte adhesion 
to brain capillaries during bacterial toxin exposure and encephalomyelitis. J Neuroinflammation 2012;9:18.

45. Kim I, Moon SO, Park SK, et al. Angiopoietin-1 reduces VEGF-stimulated leukocyte adhesion to endothelial cells by reducing ICAM-1, VCAM-1, and E-selectin expression. Circ Res 2001;89:477-9.

46. Cai Z, Falkensammer F, Andrukhov O, et al. Effects of Shock Waves on Expression of IL-6, IL-8, MCP1, and TNF-alpha Expression by Human Periodontal Ligament Fibroblasts: An In Vitro Study. Med Sci Monit 2016;22:914-21.

47. Boehler RM, Kuo R, Shin S, et al. Lentivirus delivery of IL-10 to promote and sustain macrophage polarization towards an anti-inflammatory phenotype. Biotechnol Bioeng 2014;111:1210-21.

48. Park SD, Cheon SY, Park TY, et al. Intranuclear interactomic inhibition of NF-kappaB suppresses LPSinduced severe sepsis. Biochem Biophys Res Commun 2015;464:711-7.

49. Newham P, Ross D, Ceuppens P, et al. Determination of the safety and efficacy of therapeutic neutralization of

Cite this article as: Cao H, Wang X, Zhang B, Ren M. The protective effect of vitexin in septic encephalopathy by reducing leukocyte-endothelial adhesion and inflammatory response. Ann Palliat Med 2020;9(4):2079-2089. doi: 10.21037/apm-20-1211 tumor necrosis factor-alpha (TNF-alpha) using AZD9773, an anti-TNF-alpha immune Fab, in murine CLP sepsis. Inflamm Res 2014;63:149-60.

50. Wang P, Hu Y, Yao D, et al. Omi/HtrA2 Regulates a Mitochondria-Dependent Apoptotic Pathway in a Murine Model of Septic Encephalopathy. Cell Physiol Biochem 2018;49:2163-73.

51. Chaudhry N, Duggal AK. Sepsis Associated Encephalopathy. Adv Med 2014;2014:762320.

52. Azevedo LC. Mitochondrial dysfunction during sepsis. Endocr Metab Immune Disord Drug Targets 2010;10:214-23.

53. Borghi SM, Carvalho TT, Staurengo-Ferrari L, et al. Vitexin inhibits inflammatory pain in mice by targeting TRPV1, oxidative stress, and cytokines. J Nat Prod 2013;76:1141-9.

54. Lu Y, Yu T, Liu J, et al. Vitexin attenuates lipopolysaccharide-induced acute lung injury by controlling the Nrf2 pathway. PLoS One 2018;13:e0196405.

(English Language Editor: J. Reynolds) 